\title{
COVID-19: Principais assistências de emergência e medidas de segurança aos
}

\section{profissionais de saúde}

COVIID-19: Main emergency assistance and safety measures for health professionals

COVID-19: Principales medidas de asistencia de emergencia y seguridad para los profesionales de

la salud

Heriederson Sávio Dias Moura

ORCID: https://orcid.org/0000-0001-7654-2402

Universidade de São Paulo, Brasil

E-mail: heriederson@gmail.com

Verônica Lima Araújo

ORCID: https://orcid.org/0000-0001-7809-1561 Singular Educacional, Brasil

E-mail: veronicalima29.16@gmail.com

Francisca Pereira da Silva

ORCID: https://orcid.org/0000-0002-8907-1248

Singular Educacional, Brasil

E-mail: pfrancisca463@gmail.com

Francinete Gonçalves de Oliveira

ORCID: https://orcid.org/0000-0002-2853-9636

Singular Educacional, Brasil

E-mail: francineteoliveira2008@yahoo.com.br

Raimundo Nonato Barbosa de Oliveira Filho

ORCID: https://orcid.org/0000-0003-1878-298X

Singular Educacional, Brasil

E-mail: rbfilho12@gmail.com

Diorges Boone da Silva

ORCID: https://orcid.org/0000-0001-7129-4534 Singular Educacional, Brasil

E-mail: diorges248@gmail.com

Anderson Lima Cordeiro da Silva ORCID: https://orcid.org/0000-0001-6777-0622

Universidade de São Paulo, Brasil

E-mail: andersoncordeiro@usp.br

Marinara de Mendonça Bezerra

ORCID: https://orcid.org/0000-0001-9425-0972

Centro Universitário Fametro, Brasil

E-mail: marinaramendonca@gmail.com

André Luiz Freitas Nogueira

ORCID: https://orcid.org/0000-0002-4698-8566

Secretaria Municipal de Saúde de Rio Preto da Eva, Brasil

E-mail: andreefreitas@ outlook.com

Eline Araújo de Oliveira

ORCID: https://orcid.org/0000-0003-1485-1263

Universidade Federal do Amazonas, Brasil

E-mail: elinearaujoo@icloud.com

Evelyn Barros de Oliveira

ORCID: https://orcid.org/0000-0002-2048-4052

WF Control, Brasil

E-mail: evelynbarros1996@outlook.com

Crislene da Silva de Lima

ORCID: https://orcid.org/0000-0003-0551-7298

Centro Universitário Fametro, Brasil

E-mail: crislene.slima@gmail.com

Raul dos Santos Reis

ORCID: https://orcid.org/0000-001-7354-5261

Universidade do Estado do Amazonas, Brasil

E-mail: raulreis9@gmail.com

Pedro de Oliveira Nogueira

ORCID: https://orcid.org/0000-0002-8309-5759 Universidade Paulista, Brasil 


\title{
Resumo
}

Este estudo teve como objetivo identificar na literatura científica as principais assistências no âmbito das emergências e as medidas de segurança para os profissionais de saúde durante a pandemia da COVID-19. Trata-se de uma pesquisa do tipo revisão integrativa de literatura, a partir de publicações científicas indexadas as bases de dados da Scientific Electronic Library Online (SciELO), National Library of Medicine (PUBMED) e o Google Acadêmico, cuja amostra final se deu por 21 artigos. As principais assistências de emergência prestadas durante a pandemia da COVID-19 estão os psicológicos, odontológicos e os cirúrgicos, que demonstraram redução dos casos em virtude das medidas de segurança adotadas. Na busca por minimizar as infecções a profissionais de saúde e pacientes que se encontram nos ambientes hospitalares, medidas de segurança foram adotadas, como o uso de EPI's de acordo com a complexidade de atendimento e realizado treinamento para o manuseio adequado desses equipamentos de segurança. $\mathrm{O}$ presente estudo demostrou que as assistências de emergência prestadas durante a pandemia da COVID-19 buscaram ofertar atendimentos seguros, visando resguardar a saúde dos profissionais que atuam na linha de frente, assim como dos pacientes.

Palavras-chave: Serviços de atendimento de emergência; COVID-19; Profissionais de saúde.

\begin{abstract}
This study aimed to identify in the scientific literature the main emergency assistance and safety measures for health professionals during the OVID-19 pandemic. It is an integrative literature review type research, from scientific publications indexed to the Scientific Electronic Library Online (SciELO), National Library of Medicine (PUBMED) and Academic Google databases, whose final sample was 21 articles. The main emergency assistance provided during the OVID-19 pandemic are the psychological, dental and surgical ones, which demonstrated a reduction in cases due to the safety measures adopted. In the search to minimize infections to health professionals and patients who are in hospital environments, safety measures were adopted, such as the use of IPE's according to the complexity of care and training for proper handling of these safety equipment. The present study demonstrated that the emergency assistance provided during the COVID-19 pandemic sought to offer safe care, aiming to safeguard the health of professionals who work in the front line, as well as patients.
\end{abstract}

Keywords: Emergency assistance services; COVID-19; Health professionals.

\section{Resumen}

El objetivo de este estudio era identificar en la literatura científica las principales medidas de asistencia de emergencia y seguridad para los profesionales de la salud durante la pandemia de OVID-19. Se trata de una búsqueda integradora de tipo revisión de literatura, basada en publicaciones científicas indexadas en las bases de datos Scientific Electronic Library Online (SciELO), National Library of Medicine (PUBMED) y Academic Google, cuya muestra final fue de 21 artículos. La principal asistencia de emergencia prestada durante la pandemia de OVID-19 son las de carácter psicológico, dental y quirúrgico, que demostraron una reducción de los casos debido a las medidas de seguridad adoptadas. En la búsqueda de reducir al mínimo las infecciones a los profesionales de la salud y a los pacientes en los entornos hospitalarios, se adoptaron medidas de seguridad, como el uso de EPI según la complejidad de la atención y la capacitación para el manejo adecuado de estos equipos de seguridad. El presente estudio demostró que la asistencia de emergencia prestada durante la pandemia del OVID-19 tenía por objeto ofrecer una atención segura, con el fin de salvaguardar la salud de los profesionales que trabajan en primera línea, así como la de los pacientes.

Palabras clave: Servicios de asistencia de emergencia; COVID-19; Profesionales de la salud.

\section{Introdução}

Em janeiro de 2020, a Organização Mundial da Saúde (OMS) apontou o surto da doença COVID-19 como uma Emergência de Saúde Pública de Importância Internacional (ESPII) - o mais alto nível de alerta previsto no Regulamento Sanitário Internacional. Porém, a OMS caracterizou como pandemia somente em 11 de março de 2020 (OMS, 2020a). Pandemia é o termo usado para indicar que uma epidemia se espalhou para dois ou mais continentes com transmissão contínua de pessoa para pessoa (OMS, 2020b). 
O vírus SARS-CoV-2 tem capacidade de invasão celular através da enzima conversora de angiotensina 2 (ECA2) expressas no epitélio pulmonar inferior e nas células da mucosa do intestino delgado. A transmissão ocorre quando uma pessoa infectada tem contato próximo a outra por meio de gotículas de salivas ricas com o vírus, ao aperto das mãos, espirro, tosse, catarro, como também por objetos ou superfícies contaminadas (celulares, mesas, maçanetas, brinquedos, teclados de computador, dentre outros) (Brasil, 2020).

Um levantamento feito pela OMS identificou que a maioria dos pacientes com COVID-19 (cerca de 80\%) podem ser assintomáticos e estimou que em torno de $20 \%$ dos casos podem necessitar de suporte hospitalar em decorrência de sintomatologia respiratória, destes casos aproximadamente 5\% em virtude da insuficiência respiratória (Harapan et al., 2020).

A insuficiência respiratória é o processo no qual o organismo perde a capacidade de capitar oxigênio e eliminar gás carbônico de maneira suficiente para suprir a demanda metabólica do organismo. Pacientes com infecções pela COVID-19 apresentaram falta de ar em média com oito dias e a síndrome do desconforto respiratório agudo (SDRA) com nove dias (Huang et al., 2020), os que apresentam sintomas graves da COVID-19 podem progredir rapidamente para SDRA (Chen, 2020; Holshue, 2020).

Em estudo de Huang et al. (2020), foi identificado que todos os pacientes com infecção pela COVID-19 apresentaram quadro de pneumonia, assim como algumas complicações pós-infecção (síndrome respiratória aguda grave, lesão cardíaca aguda, lesão renal aguda, choque e infecções secundárias). O estudo de Chen et al. (2020), mostrou casos de infecções secundárias por bactérias e fungos em pacientes diagnosticados com pneumonia por COVID-19, sendo essas Acinetobacter baumannii, Aspergillus flavus e Klebsiella pneumoniae (ambas em um paciente), Candida glabrata (um caso) e Candida albicans (um caso).

Atualmente, os pesquisadores apontam que a COVID-19 pode se apresentar como uma gripe por meio de sintomas brandos, além de doenças mais graves (Balleste, 2020). Porém, as manifestações mais graves são descritas em pessoas idosas com doenças pré-existentes, como hipertensão, doenças cardíacas, doenças pulmonares, câncer ou diabetes; além disso, pessoas com histórico de tabagismo estão mais vulneráveis as complicações pela COVID-19, uma vez que esses indivíduos podem ser portadores de doenças pulmonares, assim como apresentar capacidade pulmonar reduzida (OPAS, 2020).

Mundialmente, no dia 12 de janeiro de 2021, foram registrados 89.707.115 casos confirmados e 1.940 .352 mortes pela COVID-19. Destacando-se maiores concentrações no continente Europeu e nas Américas nesse período, registando juntos 68.804.610 casos confirmados da doença e 1.555.926 óbitos confirmados, sendo os dois continentes os mais afetados pela doença até este momento (OMS, 2020b). Segundo o Ministério da Saúde (MS), em 12 de janeiro de 2021 o Brasil registrou 8.195.637 casos confirmados de COVID-19, além de ter registrado 204.690 óbitos, dados estes repassados pelas Secretarias Estaduais de Saúde.

$\mathrm{Na}$ busca por melhorias na assistência aos pacientes infectados pela COVID-19, diversos grupos de pesquisas têm desenvolvido estudos voltados a redução dos casos e, consequente colaboração direta para transformação dos serviços em saúde (Marques et al., 2020). Principalmente de emergência, que no ápice das infecções pela COVID-19 apresentavam grande números de admissões de pacientes, levando as unidades hospitalares a uma lotação com casos positivos da doença (Reza et al., 2020).

Os profissionais de saúde que atuam diretamente no cuidado ao paciente estão expostos a diversos patógenos e riscos. Estudo desenvolvido por Wang et al. (2020), observou que o número de indivíduos com infecções relacionadas ao âmbito hospitalar foi considerável, dos 138 pacientes estudados, 40 (29\%) eram profissionais de saúde, sendo 31 (77,5\%) de enfermarias, 7 (17,5\%) de pronto-socorro e 2 (5\%) de UTI. A mesma pesquisa mostrou ainda que pelo menos 10 profissionais de saúde foram infectados por um paciente que teve admissão no setor de clínica cirúrgica por dores abdominais. 
Durante a pandemia da COVID-19 diversos paciente necessitaram de atendimentos de emergência, dentre esses há registros de assistências psicológicas (Sánchez, 2020), odontológicas (Sepulveda-Verdugo et al., 2020), cardiovasculares (Fernández et al., 2020), bem como outras providências inerentes a equipe e ao setor de emergência.

A recente pandemia da COVID-19 trouxe a necessidade da difusão de conhecimentos nessa área, seja na assistência quanto à segurança dos profissionais de saúde que atuam na linha de frente ao combate ao vírus, principalmente emergências. Desse modo, a presente pesquisa tem como objetivo identificar na literatura científica as medidas de segurança para o profissional e principais assistências no âmbito das emergências durante a pandemia da COVID-19.

\section{Metodologia}

O presente estudo trata-se de uma revisão integrativa sobre o tema abordado, utilizou-se desse tipo pesquisa, pois apresenta a síntese de múltiplos estudos científicos, com ferramentas que utilizam métodos rigorosos de busca e análise de achados. Além disso, possibilita obter conclusões gerais de específicas da área, corroborando para a minúcia e disseminação do conhecimento das questões analisadas e do modo que são estudadas recentemente (Mendes et al., 2008).

Trata-se de uma pesquisa descritiva, exploratório, de cunho qualitativo do tipo revisão integrativa de literatura. De acordo com Pereira et al. (2018), o método qualitativo é utilizado como um processo indutivo, o qual almeja descrever um fenômeno de estudo por meio da compreensão do pesquisador acerca de uma temática.

A partir de publicações científicas indexadas as bases de dados da Scientific Electronic Library Online (SciELO), National Library of Medicine (PUBMED) e o Google Acadêmico, com os seguintes descritores: Serviços de Atendimento de Emergência, COVID-19, Profissionais de Saúde; de acordo com o Portal de Descritores em Ciência da Saúde (DeCS).

Os critérios de inclusão foram: artigos completos, escritos nos idiomas português, inglês ou espanhol, disponíveis gratuitamente e dos últimos 5 anos (2016 a 2020). Critérios de exclusão foram: artigos em formato de resumo, monografias, dissertações de mestrado, teses de doutorado e publicações duplicadas.

Para coleta de dados dos artigos selecionados foi elaborado um documento para o registro, no qual consta os campos de autores, título do periódico, ano de publicação, revista e base de dados (Quadra 1). Após a aplicação dos critérios de inclusão e exclusão, os artigos foram lidos na íntegra, sendo assim possível realizar a extração dos fragmentos em forma de citação.

Com relação aos critérios éticos seguimos as normativas a boa conduta em pesquisa, livre de plágios e de acordo com a portaria 466/2010.

Quadro 1. Relação das publicações selecionadas para o estudo.

\begin{tabular}{|c|l|c|c|c|}
\hline AUTORES & \multicolumn{1}{|c|}{ TíTULO DO PERIÓDICO } & $\begin{array}{c}\text { ANO DE } \\
\text { PUBLICAÇÃo }\end{array}$ & REVISTA & $\begin{array}{c}\text { BASE DE } \\
\text { DADOS }\end{array}$ \\
\hline Silva et al. & $\begin{array}{l}\text { Condições de trabalho e falta de informações } \\
\text { sobre o impacto da COVID-19 entre } \\
\text { trabalhadores da saúde. }\end{array}$ & 2020 & $\begin{array}{c}\text { Revista Brasileira } \\
\text { de Saúde } \\
\text { Ocupacional }\end{array}$ & SciELO \\
\hline $\begin{array}{c}\text { Marques et } \\
\text { al. }\end{array}$ & $\begin{array}{l}\text { COVID-19: nursing care for safety in the } \\
\text { mobile pre-hospital service. }\end{array}$ & 2020 & $\begin{array}{l}\text { Texto \& Contexto } \\
\text { - Enfermagem }\end{array}$ & SciELO \\
\hline
\end{tabular}




\begin{tabular}{|c|c|c|c|c|}
\hline $\begin{array}{l}\text { Ribeiro et } \\
\text { al. }\end{array}$ & $\begin{array}{l}\text { Saúde e segurança de profissionais de saúde } \\
\text { no atendimento a pacientes no contexto da } \\
\text { pandemia de Covid-19: revisão de literatura. }\end{array}$ & 2020 & $\begin{array}{l}\text { Revista Brasileira } \\
\text { de Saúde } \\
\text { Ocupacional }\end{array}$ & SciELO \\
\hline $\begin{array}{l}\text { Sepúlveda- } \\
\text { Verdugo et } \\
\text { al. }\end{array}$ & $\begin{array}{l}\text { Consideraciones en la Atención Odontológica } \\
\text { de Urgencia en Contexto de Coronavirus } \\
\text { COVID-19 (SARS-CoV-2). }\end{array}$ & 2020 & $\begin{array}{c}\text { International } \\
\text { Journal of } \\
\text { Odontostomatolog } \\
\text { y }\end{array}$ & SciELO \\
\hline $\begin{array}{l}\text { Boin-Bakit } \\
\text { \& Melián- } \\
\text { Rivas }\end{array}$ & $\begin{array}{l}\text { La atención Odontológica a Pacientes } \\
\text { COVID-19 Positivo ¿Qué hacer ante una } \\
\text { Urgencia? }\end{array}$ & 2020 & $\begin{array}{c}\text { International } \\
\text { journal of } \\
\text { odontostomatolog } \\
\text { y }\end{array}$ & SciELO \\
\hline Reza et al. & $\begin{array}{l}\text { Emergency Surgery in Urology during the } \\
\text { COVID-19 Pandemic. }\end{array}$ & 2020 & $\begin{array}{l}\text { International } \\
\text { Brazilian Journal } \\
\text { of Urology }\end{array}$ & SciELO \\
\hline Sánchez & $\begin{array}{l}\text { La psicología de emergencias ante la COVID- } \\
\text { 19: enfoque desde la prevención, detección y } \\
\text { gestión operativa del riesgo. }\end{array}$ & 2020 & Clínica y Salud & SciELO \\
\hline Ağalar et al. & $\begin{array}{l}\text { Protective measures for COVID-19 for } \\
\text { healthcare providers and laboratory personnel. }\end{array}$ & 2020 & $\begin{array}{l}\text { Turkish Journal of } \\
\text { Medical Sciences }\end{array}$ & PubMed \\
\hline Jiang et al. & $\begin{array}{l}\text { The prevalence, characteristics, and } \\
\text { prevention status of skin injury caused by } \\
\text { personal protective equipment among medical } \\
\text { staff in fighting COVID-19: a multicenter, } \\
\text { cross-sectional study. }\end{array}$ & 2020 & $\begin{array}{l}\text { Advances in } \\
\text { Wound Care }\end{array}$ & PubMed \\
\hline Jin et al. & $\begin{array}{l}\text { Virology, Epidemiology, Pathogenesis, and } \\
\text { Control of COVID-19. }\end{array}$ & 2020 & Viruses & PubMed \\
\hline Chow et al. & $\begin{array}{l}\text { Symptom Screening at Illness Onset of Health } \\
\text { Care Personnel With SARS-CoV-2 Infection } \\
\text { in King County, Washington. }\end{array}$ & 2020 & $\begin{array}{l}\text { JAMA Network } \\
\text { Open }\end{array}$ & PubMed \\
\hline Keeley et al. & $\begin{array}{l}\text { Roll-out of SARS-CoV-2 testing for } \\
\text { healthcare workers at a large NHS Foundation } \\
\text { Trust in the United Kingdom, March } 2020 .\end{array}$ & 2020 & Euro Surveillance & PubMed \\
\hline Boa et al. & $\begin{array}{l}\text { 2019-nCoV epidemic: address mental health } \\
\text { care to empower society. }\end{array}$ & 2020 & The Lancet & PubMed \\
\hline Zhang et al. & $\begin{array}{l}\text { Survey of Insomnia and Related Social } \\
\text { Psychological Factors Among Medical Staff } \\
\text { Involved in the } 2019 \text { Novel Coronavirus } \\
\text { Disease Outbreak. }\end{array}$ & 2020 & $\begin{array}{l}\text { Frontiers in } \\
\text { Psychiatry }\end{array}$ & PubMed \\
\hline $\begin{array}{l}\text { Garcia- } \\
\text { Castrillo et } \\
\text { al. }\end{array}$ & $\begin{array}{l}\text { European Society For Emergency Medicine } \\
\text { position paper on emergency medical systems' } \\
\text { response to COVID-19. }\end{array}$ & 2020 & $\begin{array}{l}\text { European Journal } \\
\text { of Emergency } \\
\text { Medicine }\end{array}$ & PubMed \\
\hline
\end{tabular}




\begin{tabular}{|c|c|c|c|c|}
\hline Xia et al. & $\begin{array}{l}\text { Emergency Caesarean delivery in a patient } \\
\text { with confirmed COVID-19 under spinal } \\
\text { anaesthesia. }\end{array}$ & 2020 & $\begin{array}{l}\text { Journal of } \\
\text { Anesthesia }\end{array}$ & PubMed \\
\hline Ma et al. & $\begin{array}{l}\text { Emergency and essential surgical healthcare } \\
\text { services during COVID-19 in low- and } \\
\text { middle-income countries: A perspective. }\end{array}$ & 2020 & $\begin{array}{c}\text { International } \\
\text { Journal of Surgery }\end{array}$ & PubMed \\
\hline $\begin{array}{l}\text { Paganini et } \\
\text { al. }\end{array}$ & $\begin{array}{l}\text { Translating COVID-19 Pandemic Surge } \\
\text { Theory to Practice in the Emergency } \\
\text { Department: How to Expand Structure. }\end{array}$ & 2020 & $\begin{array}{l}\text { Disaster Medicine } \\
\text { and Public } \\
\text { Health Preparedne } \\
\text { ss }\end{array}$ & PubMed \\
\hline Yao et al. & $\begin{array}{l}\text { Emergency tracheal intubation in } 202 \text { patients } \\
\text { with COVID-19 in Wuhan, China: lessons } \\
\text { learnt and international expert } \\
\text { recommendations. }\end{array}$ & 2020 & $\begin{array}{c}\text { British Journal of } \\
\text { Anaesthesia }\end{array}$ & PubMed \\
\hline Yang et al. & $\begin{array}{l}\text { Clinical Characteristics of Patients With } \\
\text { Coronavirus Disease } 2019 \text { (COVID-19) } \\
\text { Receiving Emergency Medical Services in } \\
\text { King County, Washington. }\end{array}$ & 2020 & $\begin{array}{c}\text { JAMA Network } \\
\text { Open }\end{array}$ & PubMed \\
\hline $\begin{array}{l}\text { Gallasch et } \\
\text { al. }\end{array}$ & $\begin{array}{l}\text { Prevenção relacionada à exposição } \\
\text { ocupacional do profissional de saúde no } \\
\text { cenário de COVID-19. }\end{array}$ & 2020 & $\begin{array}{c}\text { Revista } \\
\text { Enfermagem da } \\
\text { Universidade } \\
\text { Estadual do Rio de } \\
\text { Janeiro }\end{array}$ & $\begin{array}{c}\text { Google } \\
\text { Acadêmico }\end{array}$ \\
\hline
\end{tabular}

Fonte: Moura et al. (2021).

Para o presente estudo foram selecionados 21 artigos, obedecendo dos critérios de inclusão e exclusão à pesquisa proposta (Quadro 1). As publicações foram subdivididas em duas categorias, a primeira aborda as principais assistências em saúde no âmbito das emergências durante a pandemia da COVID-19 e a segunda as medidas de segurança aos profissionais de saúde nas emergências durante a pandemia da COVID-19.

Para a primeira categoria foram selecionados 10 artigos e para segunda 11 publicações. Do periódico da PubMed foram selecionadas 13 (62\%) publicações, SciELO 7 (33\%) e Google Acadêmico 1 (5\%). Observou-se que os 21 estudos (100\%) inclusos nesta pesquisa são do ano de 2020, tal fato se dá devido a COVID-19 ser uma doença recente mundialmente. Contudo, há poucos conhecimentos sobre as características dos pacientes que necessitam de atendimentos de emergência (Yang et al., 2020).

\section{Resultados e Discussão}

\subsection{Principais assistências em saúde no âmbito das emergências durante a pandemia da COVID-19}

Alguns estudos mostram as características clínicas dos pacientes atendidos nos serviços de urgência e emergência, como o realizado por Yang et al., (2020), no laboratório de King County com 124 pacientes positivos para a COVID-19 que necessitaram do serviço médico de emergência apresentam idade média de 75,7 (13,2\%) anos e 66 (53,2\%) eram mulheres, 41 foram atendidos em decorrência de doenças de origem desconhecida (27,9\%), 37 com dispneia (25,2\%), 22 vítimas de trauma $(15,0 \%)$ e 19 com doenças infecciosas (12,9\%). Por sua vez, o estudo de Yao et al. (2020), demonstrou que a idade média dos pacientes da sua pesquisa era de 65 anos $(63,4 \%)$, com predomínio do sexo masculino $(67,3 \%)$. 
Em decorrência da pandemia da COVID-19 os departamentos de emergência (DE) apresentaram uma superlotação por casos suspeitos da infecção. Nesse sentido, os DE's buscaram manter suas ações voltadas a minimizar a exposição dos profissionais de saúde e pacientes suspeitos, condutas para adequar os espaços foram tomadas, como a remoção para áreas hospitalares com pouco fluxo, corredores pouco utilizados no cotidiano, criação de salas específicas, solicitação de internações imediatas e direcionamento para setores adequados aos pacientes fora de suspeita (Paganini et al., 2020).

A população em geral, incluindo os profissionais de saúde, foram afetados por diversas situações que levaram ao medo, preocupação e reações de insegurança que estão intimamente ligados à quando há risco eminente de morte ou em situações de emergência; dessa forma, o fator psicológico apresenta emoções tidas como previsíveis ao ser humano e necessitam de atendimentos de emergência, visando promover uma saúde mental aos cidadãos (Sánchez, 2020).

Além dos atendimentos psicológicos de emergência, os quais demonstram grande importância para a saúde mental da população e dos profissionais, há os atendimentos mais invasivos, como os cirúrgicos, que demonstraram redução dos casos em virtude das medidas de segurança adotadas, como a quarentena, distanciamento social e auto isolamento; contudo, alguns procedimentos cirúrgicos ainda necessitam de atendimento de emergência por indicações, podendo citar cesarianas, abdômen agudo, dissecções aórticas, urológicas, infecção de tecidos moles, dentre outras (Ma et al., 2020; Reza et al., 2020; Xia et al., 2020 ;

O estudo de Xia et al. (2020), relatou o caso de uma mulher grávida admitida no Hospital da Cruz Vermelha de Wuhan em janeiro de 2020 com febre, saturação de 92\%, movimentação fetal diminuída e oligodrâmnio, a paciente foi avaliada e recomendada cesariana de emergência. Foram realizados exames para que o procedimento cirúrgico iniciasse, dentre esses a amostra de esfregaço orofaríngeo pelo ensaio RT-PCR, a qual apresentou resultado positivo para a COVID-19, após a confirmação todas medidas de segurança do centro cirúrgico e EPI's para os funcionários foram realizadas. A raquianestesia foi a escolha para a cesariana, durante o procedimento a paciente apresentou tosse, taquicardia, agitação, disfórica e hipotensão transitória; contudo, a cirurgia foi bem-sucedida, o recém-nascido transferido para enfermaria de isolamento neonatal e a mãe para a ala de isolamento adulto.

Outros procedimentos cirúrgicos de emergência são realizados, o estudo de Reza et al. (2020), mostrou as indicações da American Confederation of Urology em casos urológicos de pacientes positivos para a COVID-19, sendo essas: retenção urinária aguda, hematúria grave, trauma geniturinário, fraturas de pênis e testículos, escroto agudo, massas escrotais e gangrena de fourniers, sendo adotadas pelos urologistas medidas de biossegurança para proteger os pacientes e profissionais de saúde.

Dentre os atendimentos de emergência na saúde, identifica-se na literatura as relações dos procedimentos odontológicos e as medidas preventivas a pacientes com a COVID-19. O estudo de Boin-Bakit e Melián-Rivas (2020), relata que os atendimentos odontológicos de emergência podem aumentar o risco de contágio a equipe odontológica ou, posteriormente, a pacientes que receberem o atendimento no mesmo ambiente, uma vez que há uma elevada chance dos procedimentos serem realizados em pacientes com a COVID-19, ou ainda em indivíduos oligoassintomáticos ou assintomáticos.

As citações do estudo supracitado ainda trazem recomendações a equipe odontológica que atender pacientes positivos para COVID-19 e/ou suspeitos, sendo o isolamento imediato umas das orientações quando houver o contato próximo sem a devida proteção com aqueles que estiverem apresentando febre, tosse, falta de ar ou outros sintomas associados a doença.

Sepúlveda-Verdugo et al. (2020), citam na sua pesquisa algumas emergências odontológicas, sendo essas a patologia inflamatória pulpar, gengivite e úlcera necrosante, trauma alveolar, pericoronite, infecções odontogênicas e complicações pósextração. Os autores ainda descrevem atendimentos que não são de emergência, mas prévios a atendimentos de terapia com bifosfonatos, transplante de órgão sólido ou medula óssea, quimio ou radioterapia. Descrevem ainda que para realizar os 
atendimentos se faz necessário um ambiente hospitalar específico com condições para realizar a assistência e uma equipe multiprofissional especializada para tomada de decisões acerca dos procedimentos.

Observando a situação dos âmbitos hospitalares, verifica-se a necessidade de uma abordagem diferenciada aos pacientes positivos para COVID-19 ou casos suspeitos. Medidas de prevenção se fazem necessárias na busca por minimizar as infecções, tendo em vista que a identificação precoce proporciona uma assistência adequada, com consequente isolamento dos casos suspeitos, para assim determinar os melhores cuidados a estes pacientes (Garcia-Castrillo et al., 2020).

\subsection{Medidas de segurança aos profissionais de saúde nas emergências durante a pandemia da COVID-19}

Os serviços hospitalares de urgências e emergências são caracterizados pela sua exacerbação nos atendimentos, fato que era perceptível mesmo antes da pandemia da COVID-19; contudo, as instituições e serviços pré-hospitalares intensificaram suas medidas preventivas e melhorias para prestar uma assistência adequada aos pacientes acometidos pela COVID-19 que buscavam atendimentos (Marques et al., 2020).

Os profissionais lidam diretamente com materiais contaminados por produtos químicos e biológicos, estando com maior susceptibilidade para contrair infecções, tendo em vista o alto risco a saúde dos profissionais de saúde, estabelecer medidas que promovam a segurança no âmbito profissional torna-se necessário a utilização equipamento de proteção individual (EPI), como máscaras faciais, máscaras cirúrgicas, máscaras n95, avental cirúrgico estéril com material impermeável descartável e mangas longas (100\% polipropileno) e punho 100\% algodão, gorros descartáveis e luvas de procedimento (Marques et al., 2020).

O uso dos EPI's mostra-se como uma ferramenta que corrobora para redução dos casos de infecções pela COVID-19 em profissionais de saúde, proporcionando um ambiente de trabalho seguro e com elevação na confiança para desenvolver a assistência necessária as pacientes que buscam o serviço de saúde (Ağalar \& Öztürk Engin, 2020). Contudo, é primordial que seja difundido aos profissionais de saúde informações a da doença da COVID-19, assim como a respeito das medidas de proteção e treinamento para o manuseio adequado dos EPI's, pontos fundamentais para a eficácia do combate a novas infecções, uma vez que há técnicas para a correta paramentação e desparamentação (Gallasch et al., 2020).

De acordo com as citações da pesquisa de Ribeiro et al. (2020), a classificação para o uso de EPI's foram descritos em três categorias relacionadas a exposição, sendo a $1^{\mathrm{a}}$ categoria: máscaras cirúrgicas, vestimentas de trabalho e capotes; $2^{\mathrm{a}}$ categoria: inclui a $1^{\mathrm{a}}$ categoria, havendo inclusão dos óculos de proteção, roupas impermeáveis e protetoras, e mudança da máscara cirúrgica pela N95; $3^{\mathrm{a}}$ categoria: inclui a $2^{\mathrm{a}}$ categoria, sendo incluído as salas com uso de pressão positiva e viseiras.

Os EPI's promovem a segurança do profissional de saúde a contrair a infecção da COVID-19. Contudo, estudos como de Jiang et al. (2020), demonstram que os EPI'S apresentam fatores que possibilitam o surgimento de lesões na pele ( $43 \%$ dos casos), relacionados principalmente ao fato do maior tempo de uso desses equipamentos, aumento da sudorese, assim como atrito e constante pressão na pele, sendo observado principalmente em profissionais que utilizam os EPI's da $3^{\mathrm{a}}$ categoria.

A infecção pela COVID-19 traz a problemática que pessoas assintomáticas ou oligossintomáticas chegam a 80\% dos casos (Jin et al., 2020). Segundo Chow et al. (2020), pacientes assintomáticas ou oligossintomáticas estão ligados intimamente a transmissão da COVID-19; porém, o uso de EPI'S pelos profissionais de saúde de apresenta como uma ferramenta para diminuição da propagação do vírus. Os pesquisadores relatam ainda medidas de identificação para possíveis casos positivos em trabalhadores, como atenção aos testes sorológicos, assim como afastamento sem condição punitiva aos acometidos.

O Sheffield Teaching Hospitals NHS Foundation Trust, no Reino Unido, iniciou no mês de março de 2020 a testagem dos profissionais de saúde com sintomas para síndrome respiratória aguda grave coronavírus 2 (SARS-CoV-2), do total de 1.533 funcionários testados, 282 (18\%) tiveram teste positivo, $1.246(81 \%)$ negativo e cinco $(<1 \%)$ apresentaram resultado indeterminado. Este estudo realizou ainda o levantamento dos profissionais aptos para permanecer nos locais de trabalho, visto 
que os trabalhadores infectados podem transmitir aos pacientes, outros profissionais e, ainda familiares que tem contato (Keeley et al., 2020).

Estudo de coorte retrospectivo realizado com 72 profissionais (médicos e enfermeiros) que atuaram na linha de frente a COVID-19 em um hospital universitário de Wuhan, esses trabalhadores apresentaram resultado positivo a doença, tal fato foi relacionado a má higienização das mãos, a elevada jornada de trabalho e a exposição dos trabalhadores do setor de alto risco (Ran et al., 2020).

O cotidiano dos profissionais de saúde está repleto de inúmeros estressores. Os trabalhadores da linha de frente ao combate a COVID-19 começaram a desenvolver diversos problemas de saúde mental, como a depressão, ansiedade e estresse (Bao et al., 2020). Estudo de Zhang et al. (2020), realizado em hospitais da china contou com a participação de 1.563 médicos, os resultados demonstraram que $73,4 \%$ dos profissionais apresentavam estresse, $50,7 \%$ depressão, $44,7 \%$ ansiedade e $36,1 \%$ insônia.

Observa-se ainda que a necessidade do distanciamento e o contato menos aproximado possível as pessoas, levam os profissionais se sentirem mais isolados, além desses fatores há sobrecarga de trabalho (Ribeiro et al., 2020) e o tempo para colocar e retirar os inúmeros EPI's, aumentando o cansaço físico e mental desses trabalhadores (Zhang et al., 2020).

Ribeiro et al. (2020), destaca em sua pesque que no Brasil a carga de trabalho para os profissionais de saúde são exaustivas, atuando com horas ampliadas, EPI's em condições e números inadequados para atender a demanda de funcionários e condições de higiene baixas, por vezes, em ambientes precários para trabalhar; assim faz-se necessário um olhar mais rigoroso para a estrutura a qual esses trabalhadores estão inseridos, bem como desenvolver políticas organizacionais que analisem a saúde e segurança dos mesmos.

O profissional de saúde, principalmente os que atuam na linha de frente, incluindo os de urgências e emergências, estão sendo afetados por diversos fatores que se exacerbaram com a pandemia da COVID-19, influenciando diretamente na assistência. Desse modo, verifica-se a necessidade de ampliar as ações de vigilância a saúde dos trabalhadores da saúde (Silva et al., 2020), acompanhando o impacto do binômio profissional-paciente na incidência dos casos da COVID-19.

\section{Considerações Finais}

As principais assistências de emergência prestadas durante a pandemia da COVID-19 foram os atendimentos psicológicos, cirurgias de extrema necessidade, como as cesarianas e procedimentos urológicos, e odontológicos, outras demandas são ofertadas nesse período, atentando para direcionamento adequada dentro do âmbito hospitalar.

Na busca por minimizar as infecções dos profissionais de saúde e pacientes, medidas de segurança foram adotadas nos ambientes hospitalares, como o uso de EPI's de acordo com a complexidade de atendimento e treinamento para o manuseio adequado desses equipamentos.

Por fim, observa-se na literatura atual a escassez de estudos voltados especificamente as emergências dos casos de pacientes acometidos pela COVID-19, principalmente os que desenvolvem os quadros graves e dão entrada nas unidades hospitalares necessitando de oxigênio suplementar, ou ainda, evoluindo rapidamente para utilização da ventilação mecânica. Dessa forma, esta pesquisa permitirá que estudos futuros possam vir elucidar essas lacunas e demais pertinentes a temática abordada.

\section{Referências}

Ağalar, C., \& Öztürk Engin, D. (2020). Protective measures for COVID-19 for healthcare providers and laboratory personnel. Turk J Med Sci, 50(SI-1): 57884.

Balleste, R. (2020). El laboratorio en el diagnóstico de COVID-19 en Uruguay: resultados y desafíos. Rev. Méd. Urug., Montevideo, 36(3), 1-8. 
Bao, Y., Sun, Y., Meng, S., Shi, J. \& Lu, L. (2020). 2019-nCoV epidemic: address mental health care to empower society. The Lancet. 395(0224): e37-38. Brasil. Ministério da Saúde. Coronavírus (COVID-19): Sobre a doença - Brasília: Ministério da Saúde.

Chen, N., Zhou, M., Dong, X., Qu, J., Gong, F., Han, Y. et al. (2020). Epidemiological and clinical characteristics of 99 cases of 2019 novel coronaviruspneumonia in Wuhan, China: a descriptive study. The Lancet, 395, 507-513.

Chow, E. J., Schwartz, N. G., Tobolowsky, F. A. et al. (2020). Symptom screening at illness onset of health care personnel with SARS-CoV-2 infection in King County, Washington. JAMA. 323(20), 2087-9.

Fernandez, A., Pena, J. A. \& Mombelli, A. (2020). COVID-19 con afectación cardiovascular. Reporte de un caso. Rev. Urug. Cardiol., Montevideo, $35(2), 256-262$

Gallasch, C. H., Cunha, M. L., Pereira, L. A. S. \& Silva-Junior, J. S. (2020). Prevenção relacionada à exposição ocupacional do profissional de saúde no cenário de COVID-19. Rev Enferm UERJ, 28, e49596.

Garcia-Castrillo, L., Petrinob, R., Leachc, R., Dodtd, C., Behringere, W., Khouryf, A. \& Sabbe, M. (2020). European Society For Emergency Medicine position paper on emergency medical systems' response to COVID-19. Eur J Emerg Med., 4: 10.1097/MEJ.0000000000000701.

Harapan, H., Itoh, N., Yufika, A., Winardi, W., Keam, S., Te, H. et al. (2020). Coronavirus disease 2019 (COVID-19): A literature review. Journal of infection and Public Health, 1(Iii), 1-26.

Holshue, M. L., Holshue, M. L., DeBolt, C., Lindquist, S., Lofy, K. H., Wiesman, J., Bruce, H. et al. (2020). First case of 2019 novel coronavirus in the United States. The New England Journal of Medicine, 382, 10.

Huang, C., Wang, Y., Li, X., Ren, L., Zhao, J., Hu, Y. et al. (2020). Clinical features of patients infected with 2019 novel coronavirus in Wuhan, China. The Lancet, 395, 497-506

Jiang, Q., Song, S., Zhou, J., Liu, Y., Chen, A., Bai, Y. et al. (2020). The prevalence, characteristics, and prevention status of skin injury caused by personal protective equipment among medical staff in fighting COVID-19: a multicenter, cross-sectional study. Adv Wound Care, 9(7), 357-64.

Jin, Y., Yang, H., Ji, W., Wu, W., Chen, S., Zhang, W. \& Duan, G. (2020). Virology, epidemiology, pathogenesis, and control of covid-19. Viruses, 12(4), 372 .

Keeley, A. J., Evans, C., Colton, H., Ankcorn, M., Cope, A., State, A. et al. (2020). Roll-out of SARS-CoV-2 testing for healthcare workers at a large NHS Foundation Trust in the United Kingdom, March 2020. Euro Surveill, 25(14), 2000433.

Ma, X., Vervoort, D., Reddy, C. L., Park, K. B. \& Makasa, E. (2020). Emergency and essential surgical healthcare services during COVID-19 in low- and middle-income countries: A perspective. International Journal of Surgery, 79, 43-46.

Marques, L.C., Lucca, D. C., Alves, E. O., Fernandes, G. C. M. \& Nascimento, K. C. (2020). COVID-19: nursing care for safety in the mobile pre-hospital service. Texto contexto - enferm, 29, e20200119.

Mendes, K. D. S., Silveira, R. C. C. P. \& Galvão, C. M. (2008). Revisão integrativa: método de pesquisa para a incorporação de evidências na saúde e na enfermagem. Texto Contexto Enferm, 17(4), 758-64.

OMS. Organização Mundial de Saúde. (2020a). Relatório de situação de doença coronavírus 2019 (COVID-19) - 81. https://www.who.int/docs/defaultsource/coronaviruse/situation-reports/20200413-sitrep-84-covid-19.pdf.

OMS. Organização Mundial de Saúde. (2020b). Coronavirus disease 2019. World Health Organization, Washinton, 2633. https://covid19.who.int/.

OPAS. Organização Pan-Americana da Saúde. (2020). Folha informativa - COVID-19 (doença causada pelo novo coronavírus). https://www.paho.org/bra/index.php?option=com_content $\&$ view=article\&id=6101: covid19\&Itemid=875.

Paganini, M., Conti, A., Weinstein, E., Corte, F. D. \& Luca Ragazzoni, L. (2020). Translating COVID-19 Pandemic Surge Theory to Practice in the Emergency Department: How to Expand Structure. Disaster Med Public Health Prep., 27, 1-10.

Pereira, A. S., Shitsuka, D. M., Parreira, F. J. \& Shitsuka, R. (2018). Metodologia da pesquisa científica. Ed. UAB/NTE/UFSM. https://repositorio.ufsm.br/bitstream/handle/1/15824/Lic_Computacao_Metodologia-Pesquisa-Cientifica.pdf?sequence=1 .

Ran, L., Chen, X., Wang, Y., Wu, W., Zhang, L. \& Tan, X. (2020). Risk Factors of Healthcare Workers with Corona Virus Disease 2019: A Retrospective Cohort Study in a Designated Hospital of Wuhan in China. Clin Infect Dis, ciaa287.

Reza, M. T., Autrán-Gómez, A. M., Tardío, G. U., Bolaños, J. A. \& Rivero, J, C. G. (2020). Emergency Surgery in Urology during the COVID-19 Pandemic. Int. brazj urol., Rio de Janeiro, 46(1), 201-206.

Ribeiro, A. P., Oliveira, G. L., Silva, L. S. \& Souza, E. R. (2020). Saúde e segurança de profissionais de saúde no atendimento a pacientes no contexto da pandemia de Covid-19: revisão de literatura. Rev. bras. saúde ocup, 45, e25.

Sanchez, J. I. R. (2020). La psicología de emergencias ante la COVID-19: enfoque desde la prevención, detección y gestión operativa del riesgo. Clínica y Salud, Madrid, 31(2), 115-118.

Sepulveda-Verdugo, C., Álvarez, A. S. \& Donoso-Hofer, F. (2020). Consideraciones en la Atención Odontológica de Urgencia en Contexto de Coronavirus COVID-19 (SARS-CoV-2). Int. J. Odontostomat, Temuco, 14(3), 279-284.

Silva, L. S., Machado, E. L., Oliveira, H. N. \& Ribeiro, A. P. (2020). Condições de trabalho e falta de informações sobre o impacto da COVID-19 entre trabalhadores da saúde. Rev. bras. saúde ocup, 45, e24. 
Research, Society and Development, v. 10, n. 1, e48910112004, 2021

(CC BY 4.0) | ISSN 2525-3409 | DOI: http://dx.doi.org/10.33448/rsd-v10i1.12004

Wang, D., Hu, B., Hu, C., Zhu, F., Liu, X., Zhang, J. et al. (2020). Clinical Characteristics of 138 Hospitalized Patients With 2019 Novel CoronavirusInfected Pneumonia in Wuhan, China. JAMA. 323 (11), 1061-1069.

Xia, H., Zhao, S., Wu, Z., Luo, H., Zhou, C. \& Chen, X. (2020). Emergency Caesarean delivery in a patient with confirmed COVID-19 under spinal anaesthesia. J Anaesth., 124 (5), e216-218.

Yang, B. Y., Barnard, L. M., Emert, J. M., Drucker, C., Schwarcz, L., Counts, C. R. et al. (2020). Clinical Characteristics of Patients With Coronavirus Disease 2019 (COVID-19) Receiving Emergency Medical Services in King County, Washington. JAMA Network Open., 3(7), e2014549

Yao, W., Wang, T., Jiang, B., Gao, F., Wang, L., Zheng, H. et al. (2020). Emergency tracheal intubation in 202 patients with COVID-19 in Wuhan, China: lessons learnt and international expert recommendations. British Journal of Anesthesia., 125(1), e28-37.

Zhang, C., Yang, L., Liu, S., Ma, S., Wang, Y., Cai, Z. et al. (2020). Survey of insomnia and related social psychological factors among medical staff involved in the 2019 novel coronavirus disease outbreak. Frontiers in Psychiatry. 11(306), 1-9. 